\section{Generating awareness about enamel erosion}

Delegates attending the British Dental Conference and Dentistry Show this May in Birmingham were enriched by accredited 20 minute CPD lectures which provided a new perspective about enamel erosion, its diagnosis, prevention and care.

Presenters from Unilever's research and development/science department may have concerned some delegates with a statistic that $80 \%$ of adults present with signs of enamel erosion ${ }^{1}$ and the consequences for their oral health can be far reaching - at worst leading to tooth destruction. Yet this is a preventable condition and the dental professionals were reassured that by identifying people at risk through the BEWE Index, they can deliver education about lifestyle changes and preventive strategies to thwart patients' progressive enamel loss.

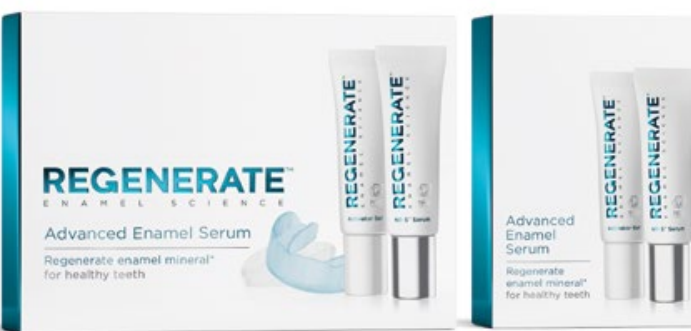

It was explained that patients' lifestyle choices can be responsible for tooth erosion, and that enamel erosion and acid attacks are the cause of up to $80 \%$ of dental problems. Enamel wear has three principal causes:

- Erosion, which is caused by dentally aggressive acid attacks

- Attrition, where teeth are worn by bruxism

- Abrasion, some of the causes are excessive use of the toothbrush, or by brushing at the wrong time - primarily too soon after meals.

The consequences of erosion include:

- Yellowing: Teeth can become yellower as a consequence of enamel thinning because the underlying dentine becomes more visible

- Transparency: It is not uncommon in the early stages of tooth erosion for the teeth to have a sand blasted look or for the tips of the front teeth to look transparent

- Sensitivity: As a consequence of the loss of enamel, the underlying dentine can become exposed. This can result in feeling sharp twinges, discomfort or pain when eating sweets, hot or cold food and drinks

- Damage: In the advanced stages, microscopic indentations arise on the surface of the teeth. If tooth erosion continues, the edges of the teeth can start to crack and have a rough feeling.

\section{Inspired ground breaking science}

The delegates were then taken through the ground-breaking science behind REGENERATE ENAMEL SCIENCE that contains NR-5 Technology, developed after over ten years' research into enamel remineralisation inspired by the elements used in bone repair treatment. The two key NR-5 ingredients, calcium silicate and sodium phosphate salts, combine to form a fresh supply of enamel minerals. The resulting dental system is the first regime capable of regenerating enamel mineral ${ }^{*}$ with exactly the same mineral from which tooth enamel is made.

The Regenerate system acts on early invisible stages of enamel erosion and helps to regenerate enamel by restoring its mineral content and micro hardness with regular use. The system is comprised of a combination of a toothpaste and serum and in vitro tests measuring enamel hardness show that it is clinically proven to regenerate enamel mineral. Eighty-two percent of enamel mineral is regenerated after three days.*

The presenters explained that dental enamel erosion occurs every day with the intake of acidic foods and drinks, yet often remains unnoticed until more advanced, with signs of tooth transparency, chipping, yellowing or increased sensitivity. Unfortunately, once enamel is lost the body cannot restore it. Regenerate Advanced Toothpaste and Advanced Serum has been clinically proven to help regenerate enamel by restoring its mineral content and micro hardness with regular use. However, the important word is 'regenerate. The treatment cannot replace lost enamel, but it can help restore eroded enamel mineral.

To boost the benefits of Regenerate Enamel Science, dental professionals were recommended to advise patients to:

- Refrain from brushing your teeth immediately after drinking acidic food or drink. Wait at least 30 minutes to an hour

- Minimise the amount of acidic food and drink consumed

- Rinse your mouth with a glass of water after consuming acidic food or drink. This helps to stabilise your mouth's natural $\mathrm{pH}$ balance

- Have regular dental check-ups and talk to your dentist or hygienist about any concerns.

A series of accredited CPD modules for the dental care team have been developed on enamel erosion, erosive tooth wear and diagnosis. Regenerate Enamel Science CPD modules are free and can be used as verified CPD hours according to the GDC guidelines. These are accessed via: https://www.regeneratenr5.co.uk/pages/professional.

Unilever has partnered the Erosive Tooth Wear Foundation, which promotes good health of the public by raising awareness and advancing education of the condition of enamel erosion and its prevention and treatment. More information about this can be found here: https://www.erosivetoothwear.com/ .

Steph Cook, Regenerate's Country Manager for the UK commented 'A commonly recurring question throughout the two days of lectures was where patients could purchase Regenerate. Regenerate Enamel Science is widely available from retailers such as Boots, Amazon and Selfridges. Find out more on our website: https://www.regeneratenr5. co.uk/collections/shop-our-products ?

\section{References}

1. Regenerate Enamel Science. Enamel erosion, explained. Available at: https://www. regeneratenr5.co.uk/pages/enamel-erosion (accessed May 2019).

*Acts on early invisible stages of enamel erosion. Helps to regenerate enamel by restoring its mineral content and micro hardness with regular use. Clinically proven. 